\title{
Elastic image registration of 2-D gels for differential and repeatability studies
}

\author{
Carlos O. S. Sorzano ${ }^{1}$, Ignacio Arganda-Carreras², Philippe Thévenaz ${ }^{3}$, Ana Beloso ${ }^{4}$, \\ Gracia Morales ${ }^{4}$, Israel Valdés ${ }^{4}$, Carmen Pérez-García ${ }^{5}$, Carmen Castillo ${ }^{5}$, \\ Elisa Garrido ${ }^{5}$ and Michael Unser ${ }^{3}$ \\ ${ }^{1}$ Bioengineering Laboratory, University San Pablo - CEU, Madrid, Spain \\ 2 Department of Computer Science, Universidad Autónoma de Madrid, Madrid, Spain \\ ${ }^{3}$ Biomedical Imaging Group, École polytechnique fédérale de Lausanne (EPFL), Switzerland \\ ${ }^{4}$ Proteomics Group, National Center of Biotechnology (CSIC), Madrid, Spain \\ ${ }^{5}$ Department of Pharmacology, Pharmaceutical Technology and Development, \\ University San Pablo - CEU, Madrid, Spain
}

One of the main applications of electrophoretic 2-D gels is the analysis of differential responses between different conditions. For this reason, specific spots are present in one of the images, but not in the other. In some other occasions, the same experiment is repeated between 2 and 12 times in order to increase statistical significance. In both situations, one of the major difficulties of these analysis is that 2-D gels are affected by spatial distortions due to run-time differences and dye-front deformations, resulting in images that are significantly dissimilar not only because of their content, but also because of their geometry. In this technical brief, we show how to use free, state-of-the-art image registration and fusion algorithms developed by us for solving the problem of comparing differential expression profiles, or computing an "average" image from a series of virtually identical gels.

\section{Keywords:}

2-D gels / Electrophoresis / Image registration / Spot matching
Received: May 21, 2007

Revised: September 18, 2007 Accepted: September 25, 2007
2-D electrophoresis is a procedure to separate and identify the proteins expressed by an organ, tissue, or cell, at a given time and under certain conditions [1]. Proteins are first separated on the basis of their $\mathrm{pI}$ by IEF in an IPG acrylamide strip. Then, proteins are separated in a perpendicular direction according to their mass by electrophoresis in a polyacrylamide gel in the presence of SDS (SDS-PAGE). Finally, a dye is applied with a selective affinity toward specific components of the proteins. The result is a bidimensional image in which small spots reveal the presence of those proteins that showed affinity to the dye and that had a specific mass and charge in the initial solution.

Correspondence: Dr. Carlos Óscar Sánchez Sorzano, Escuela Politécnica Superior, University San Pablo - CEU, Campus Urb. Montepríncipe s/n, 28668 Boadilla del Monte, Madrid, Spain E-mail: coss.eps@ceu.es

Fax: +34-91-372-4049
One of the problems of this technique is that, when comparing two different gels of similar biological content, there are important local spatial deformations that must be corrected if we want to identify biological differences or to compute an average gel from a series of biologically identical experiments. This geometrical correction is known in image processing as image registration.

Solving the registration problem can be done either by the help of landmarks [2] or the image content [3-7]. We reported in ref. [6] such preliminary results using an algorithm based on vector splines developed by us [8]. This algorithm had a number of advantages over other image-based approaches such as versatility of the estimated deformation field, accurate image interpolation performed during the estimation of the deformation field, possibility of including both image information and landmarks at the same time, and possibility to impose smoothness to the deformation field through its divergence and rotational. Our registration 
algorithm is based on the use of cubic B-splines to represent the deformation field (this allows us to represent any deformation continuous deformation field simply by reducing the spacing between splines). The algorithm minimizes in an efficient way (Levenberg-Marquardt minimization enhanced by a BFGS estimate of the local Hessian of the goal function) the quadratic error between a target image and a deformed source image. The deformation field can be constrained to be regular by penalizing its divergence and curl. Although the use of landmarks is not compulsory, in difficult cases (with very different image content) they can be very helpful to identify the correct image deformation.

In ref. [9], we extended our method to compute "quasiinvertible" deformation fields so that image A can be mapped onto image B and vice versa. (The algorithm called bUnwarpJ can be freely downloaded from http://biocomp.cnb.uam.es/ \%7eiarganda/bUnwarpJ.) This helps the optimizer to reduce the chance of getting trapped in a local minimum and opens the door to the simultaneous registration of any number of images.

In this technical brief, we show how to use bUnwarpJ to compare gels with different expressions and how to produce a single gel from a series of virtually identical gels. We show the applicability of our registration algorithm with 2-D gels used in the study of the Pseudomonas putida global Crc regulator. This protein is a global regulator of carbon metabolism in Pseudomonas. In ref. [10], the proteome profile of a $P$. putida strain was compared to that of an isogenic derivative in which the $\mathrm{crc}$ gene had been inactivated. Among others, the results showed that $\mathrm{Crc}$ is involved in the catabolic repression of $h p d$ and $h m g A$ genes from the homogentisate pathway, one of the central catabolic pathways for aromatic compounds. Based on four gels, a change in the expression of 13 proteins of the metabolic pathway was identified [10]. We registered the same gels and checked our results against those of [10]. We show in Fig. 1 the registration outcome as an RGB image, with one image assigned to the red channel and the other to the green channel. We used the same numbers as in the original paper to label the 13 spots identified in ref. [10]. As can be seen, all 13 proteins can be properly and easily identified in our registration.

Spots 5 and 6 are particularly interesting because they do not correspond to an inhibition process but to an overexpression. These two spots appear with an orange tint in Fig. 1, meaning that the registration algorithm correctly found the corresponding spots in the other image. We conclude that every protein with differential expression patterns that was identified in ref. [10] has been correctly picked out by our algorithm, too. We also picked out additional proteins that, according to our registration method, exhibit a differential expression pattern. Those, however, were not selected in ref. [10] because they were unrelated to the metabolic pathway under study.

We created 2-D gels from the cerebellum of rat brains to perform a repeatability study and to produce a single "average" gel. Proteins were first separated by IEF in a IPG strip (17 cm, pH 3-10 nonlinear) and then by SDS-PAGE in 12\% polyacrilamide gels $\left(180 \times 200 \times 1 \mathrm{~mm}^{3}\right)$. Finally, the gels were stained with silver nitrate. We used the PROTEAN Plus Dodeca Cell (BioRad) for the SDS-PAGE and the Dodeca Stainer (BioRad) for the silver stain; those devices can run and stain 12 gels at a time, which ensures that gels are processed under the same conditions and improves reproducibility.

We used the software to mutually align all pairs of images. Then, we took one of them as reference and produced the corresponding warped images of the others. Instead of computing the pointwise arithmetic average, we followed an image fusion approach to retain as much information as possible from the original images [10]. Image fusion combines the information from a set of images by keeping the most salient regions from each image under the hypothesis that information must show high local energy, which is met
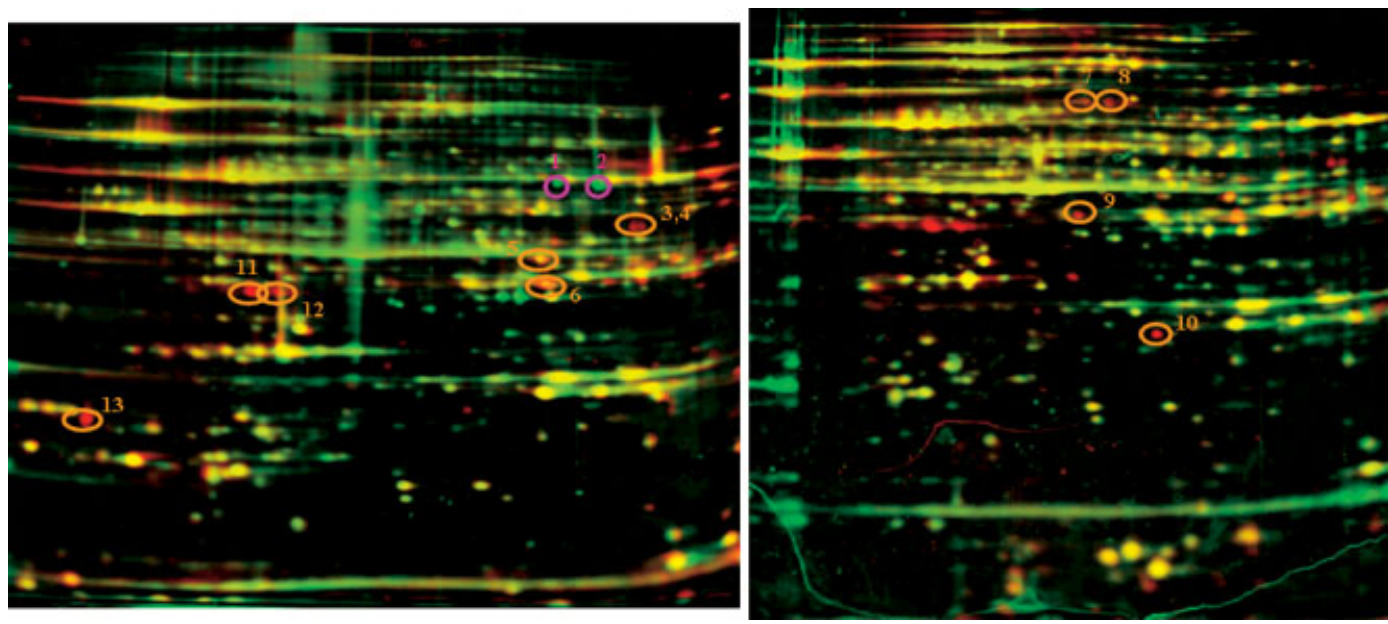

Figure 1. Registration of two pairs of gels of $P$. putida. For each pair, one of the images was put in the red channel of an RGB color image and the other one was put in the green channel. Yellow spots correspond to proteins that are equally expressed in both gels. Red spots ( 3 and $4,7-13$ ) correspond to proteins that were expressed in the first gel but not in the second. Conversely, green spots (1 and 2$)$ correspond to proteins expressed in the second gel but not in the first. Orange spots ( 5 and 6$)$ correspond to overexpressed proteins. 

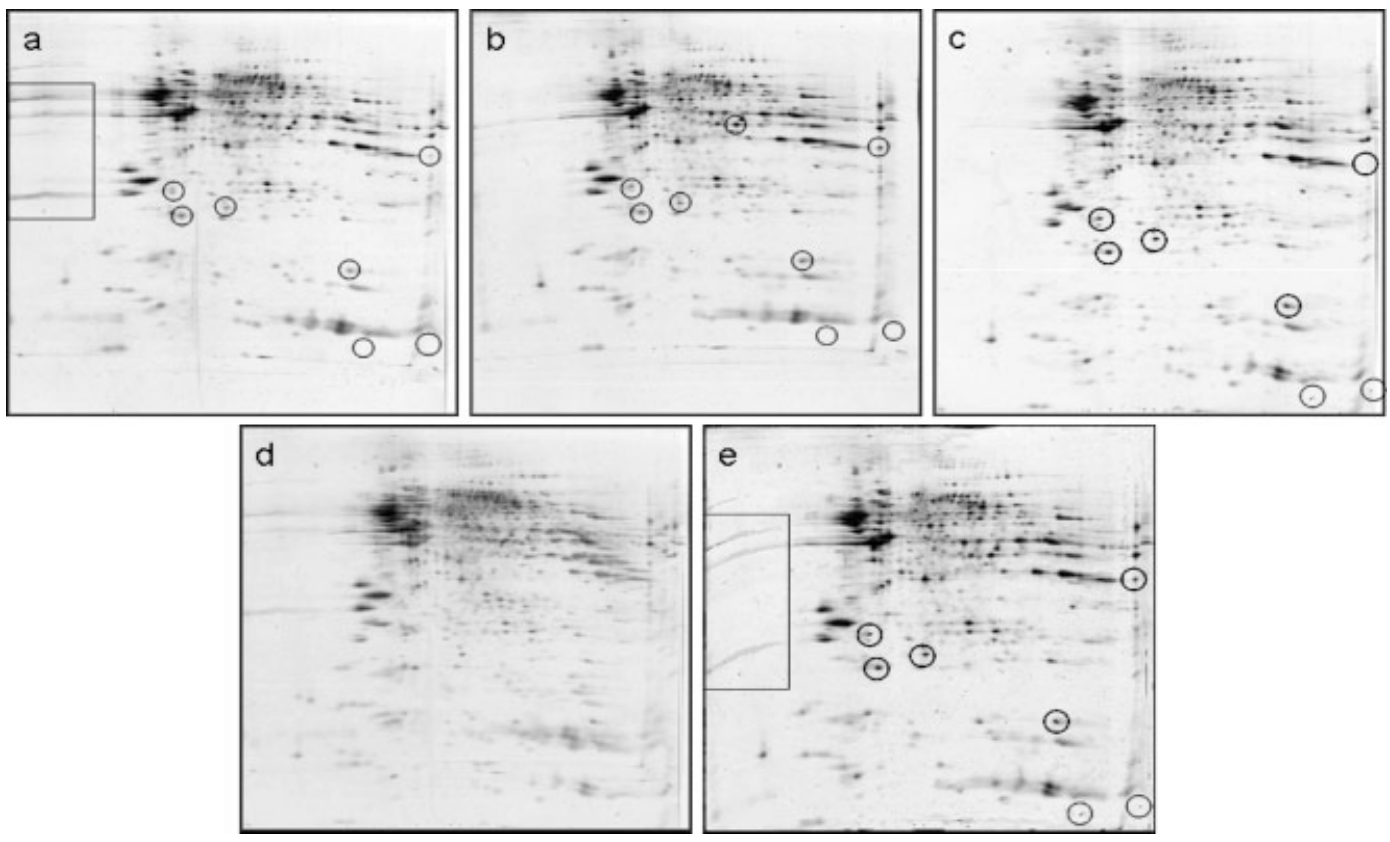

Figure 2. (a-c) Three 2-D gels with identical biological content, taken under similar conditions; (d) arithmetic average before alignment; (e) image fusion after alignment by bUnwarpJ. Despite the physical handling of multiple gels at a time, which improves repeatability, we see in (d) that some spots still do not coincide. Image registration and fusion are required to obtain (e), where we observe a much better resolved average gel combining the information present in all three gels.

by a series of aligned 2-D gels. Saliency is measured in the wavelet domain: at each location the most salient wavelet coefficient is the one with highest module (wavelet coefficients are complex numbers). However, the most salient wavelet coefficient need not be selected, instead we use this concept in combination with two consistency checks: first, if two out of three corresponding sub-band coefficients are attributed to the same image, then the third one is taken from that image, too; second, if the majority of neighboring numbers in a $3 \times 3$ window in the final wavelet map are from a different image, then we take the "outlier" coefficient also from that image. Finally, an inverse wavelet transform is performed to reconstruct the image. The software for image fusion can be freely downloaded from http://bigwww.epfl.ch/demo/edf. We show in Fig. 2 the results of a simple average of the three images and the fused image after "quasi-invertible" elastic registration. The fused image does not show multiple spots thanks to the elastic alignment and keeps the spots from the image where they are more salient thanks to the image-fusion algorithm. This produces a fused image in which the spots are as contrasted and resolved as possible (the median of the absolute difference between the fused image and the three images is 3.8\%). As a drawback undesired lines (like the ones in the rectangle of Fig. 2a) are also kept in the fused image. These lines are distorted because they have to be mapped onto flat regions with no features, and the distorted lines fit equally in any orientation. If protein spots were available around, the regularity and continuity of the deformation field would prevent too much distortion [6].
The two experiments reported in this paper show that free, open-source, publicly available, state-of-the-art imageprocessing algorithms for image registration and fusion can be used to compare gels with differential expression profiles and to build a single "average" gel from a series of virtually identical gels. This can be done in a low cost PC or Mac with a computational cost of less than 3 or 4 min.

The authors have declared no conflict of interest.

\section{References}

[1] Gorg, A.,Weiss, W., Dunn, M., Current two-dimensional electrophoresis technology for proteomics. Proteomics 2004, 4, 3665-3685.

[2] Raman, B., Cheung, A., Marten, M. R., Quantitative comparison and evaluation of two commercially available, twodimensional electrophoresis image analysis software packages, Z3 and Melanie. Electrophoresis 2002, 23, 2194-2202.

[3] Gustafsson, J. S., Blomberg, A., Rudemo, M., Warping twodimensional electrophoresis gel images to correct for geometric distortions of the spot pattern. Electrophoresis 2002, 23, 1731-1744.

[4] Rohr, K., Cathier, P., Worz, S., Elastic registration of electrophoresis images using intensity information and point landmarks. Pattern Recognit. 2004, 37, 1035-1048.

[5] Smilansky, Z., Automatic registration for images of two-dimensional protein gels. Electrophoresis 2001, 22, 1616-1626. 
[6] Sorzano, C. O. S., Thévenaz, P., Valdés, I., Beloso, A., Unser, M., Elastic image registration with applications to proteomics. Proceedings of the 5th International Workshop on Information Optics 2006, pp. 300-309.

[7] Veeser, S., Dunn, M. J., Yang, G. Z., Multiresolution image registration for two-dimensional gel electrophoresis. Proteomics 2001, 1, 856-870.

[8] Sorzano, C. O. S., Thévenaz, P., Unser, M., Elastic registration of biological images using vector-spline regularization. IEEE Trans. Biomed. Eng. 2005, 52, 652-663.
[9] Arganda-Carreras, I., Sorzano, C. O. S., Marabini, R., Carazo, J. M. et al., Consistent and elastic registration of histological sections using vector-spline regularization. Lect. Notes Comput. Sci. 2006, 4241, 85-95.

[10] Morales, G., Linares, J. F., Beloso, A., Albar, J. P. et al., The Pseudomonas putida $\mathrm{Crc}$ global regulator controls the expression of genes from several chromosomal catabolic pathways for aromatic compounds. J. Bacteriol. 2004, 186, 1337-1344. 